\title{
Discovery of the Third Ambient Aspirin Polymorph
}

\section{Chunhua Tony $\mathrm{Hu}$}

Department of Chemistry, New York University, 100 Washington Square East, New York, New York 10003 (USA). E-mail: chunhua.hu@,nyu.edu

McCrone stated in 1965, "Every compound has different polymorphic forms, and that, in general, the number of forms known for that compound is proportional to the time and money spent in research on that compound." It holds absolutely true for polymorphism of aspirin, one of the most widely consumed medications for pain relief. Since its invention in the late 1890s, aspirin remains only one crystal form until 2005. Thereafter, the second polymorph (II), similar in structure to the form I, was reported. In March of 2012, when examining the crystallization of aspirin from the melt, my colleague Alexander Shtukenberg discovered the concomitant crystallization of banded spherulites of form I and smooth spherulites of an unknown form (see picture). Because of the coexistence of form $\mathbf{I}$ and the new form, and the metastablity of this new form at the room temperature, there was a big headache to separate the new form. In addition, we were facing the challenges of obtaining its high-quality diffraction data and determining its crystal structure. Eventually, after the five-year not-giving-up effort and with a great teamwork using a combination of X-ray powder diffraction analysis and crystal structure prediction algorithms, we successfully solved the crystal structure of the third ambient polymorph of aspirin (namely form IV in consideration of the high-pressure form III transformed from form I).

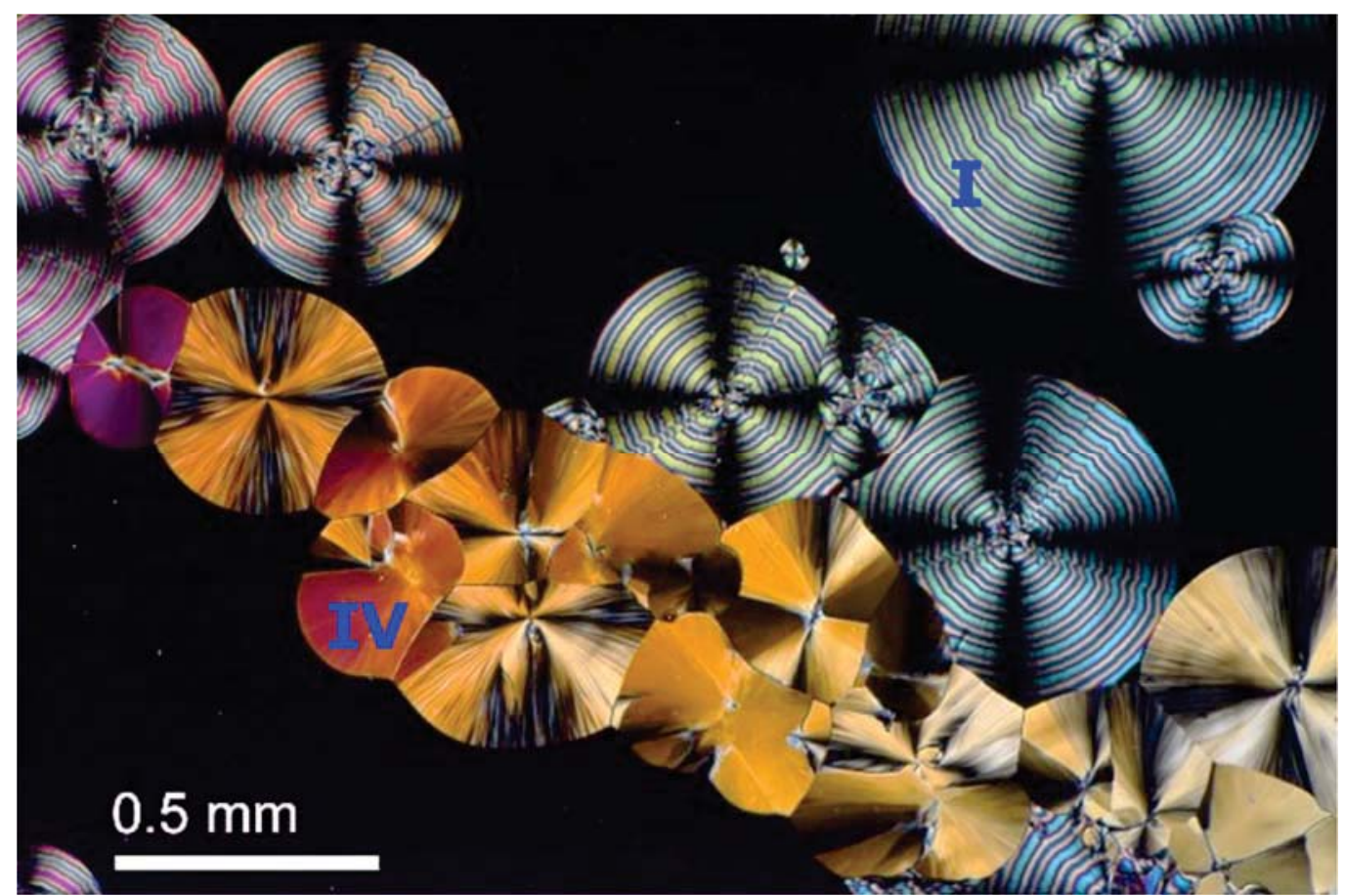

